\title{
Salary Information for Nuclear Engineers and Health Physicists, July 1996
}

\author{
Prepared by: \\ Analysis and Evaluation Programs \\ Oak Ridge Institute for Science and Education \\ Prepared for: \\ Office of Personnel \\ U.S. Nuclear Regulatory Commission
}

October 1996

This document describes activities performed under Contract Number DE-AC05-760R00033 between the U.S.

Department of Energy and Oak Ridge Associated Universities under interagency agreement with the U.S. Nuclear Regulatory Commission.

All opinions expressed in this paper are the authors' and do not necessarily reflect policies and views of the U.S. Department of Energy or the Oak Ridge Institute for Science and Education. 


\section{DISCLAIMER}

This report was prepared as an account of work sponsored by an agency of the United States Government. Neither the United States Government nor any agency thereof, nor any of their employees, make any warranty, express or implied, or assumes any legal liability or responsibility for the accuracy, completeness, or usefulness of any information, apparatus, product, or process disclosed, or represents that its use would not infringe privately owned rights. Reference herein to any specific commercial product, process, or service by trade name, trademark, manufacturer, or otherwise does not necessarily constitute or imply its endorsement, recommendation, or favoring by the United States Government or any agency thereof. The views and opinions of authors expressed herein do not necessarily state or reflect those of the United States Government or any agency thereof. 


\section{DISCLAIMER}

Portions of this document may be illegible in electronic image products. Images are produced from the best available original document. 


\section{Table of Contents}

Introduction

Summary Highlights $\quad$................................................................................ 2

Salary Tables

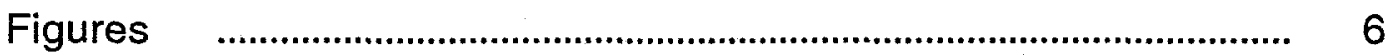

Survey Respondents ............................................................................... 14

\section{List of Tables}

1. Salary Information Requests and Responses, July $1996 \quad \ldots . . . . . . . . \quad 1$

2. Utilities' Salary Information, July $1996 \quad$.............................................. 3

3. Non-Utilities' Salary Information, July $1996 \quad$.................................... 4

4. Annual Salary Percentage Changes, 1992-1996 …………......... 5

\section{List of Figures}

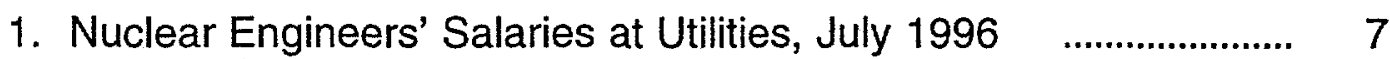

2. Nuclear Engineers' Salaries at Non-Utilities, July $1996 \quad \ldots \ldots \ldots . . . . . \quad 8$

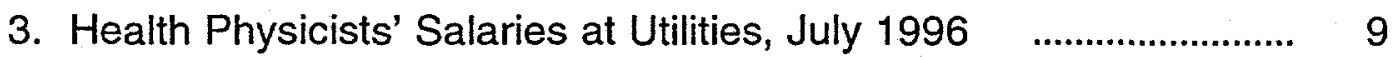

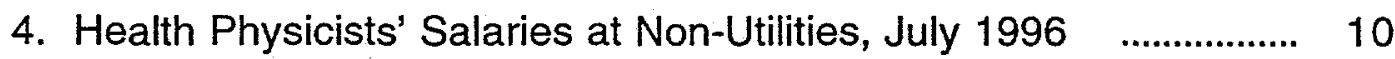

5. B.S. Level, Zero Experience, Salary Percentage Changes $\quad$........ 11

6. M.S. Level, Zero Experience, Salary Percentage Changes $\quad$........ 12

7. Ph.D. Level, Zero Experience, Salary Percentage Changes $\quad \ldots . . . .13$ 


\section{INTRODUCTION}

Salary information was collected for July 1996 for personnel working as nuclear engineers and health physicists. The salary information includes personnel at the B.S., M.S., and Ph.D. levels with zero, one, three, four to seven, and eight to ten years of professional work experience.

Information is provided for utilities and non-utilities. Non-utilities include private sector organizations and U.S. Department of Energy contractor-operated facilities. Government agencies, the military, academic organizations, and medical facilities are excluded. In previous years the salary data have been collected for October. In 1996, the data were collected for July; thus, some caution must be exercised in making annual salary trend comparisons.

The number of requests for information and of responses received are summarized in Table 1.

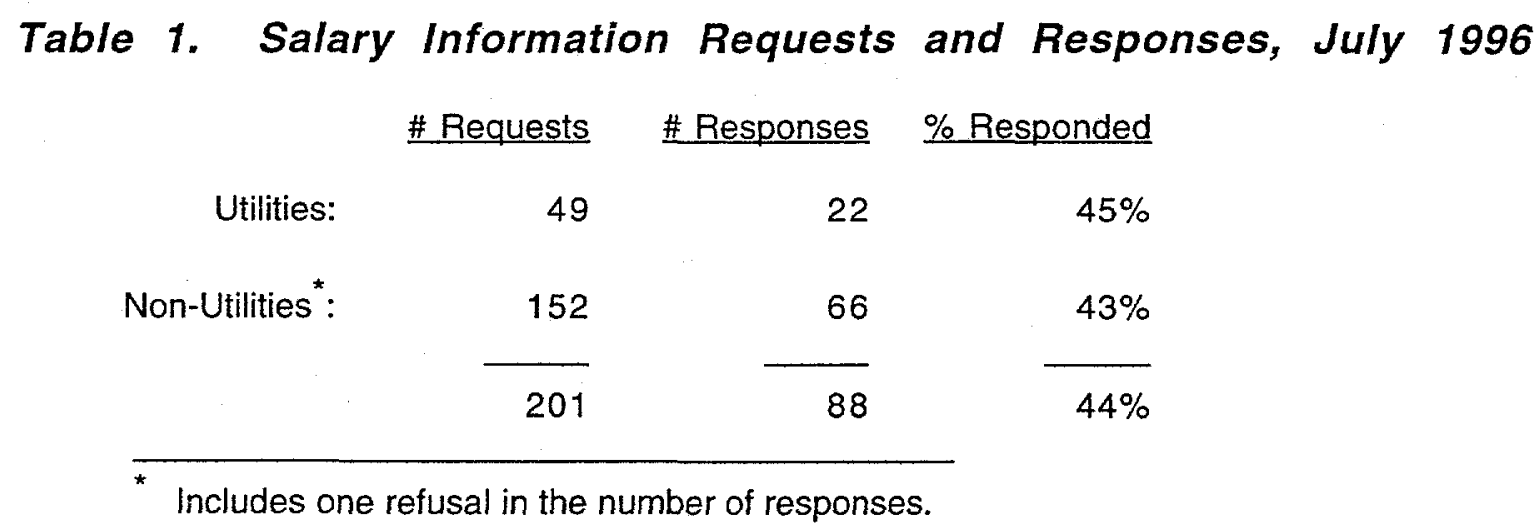




\title{
SUMMARY HIGHLIGHTS -- JULY 1996 SALARY INFORMATION
}

\section{Salaries for Nuclear Engineers (see Table 2 and 3)}

\author{
Nuclear engineers' starting salaries reported by utilities and non-utilities
}

For B.S. level with zero years of professional work experience, the average annual salary is $\$ 37,100$ for utilities and $\$ 36,700$ for non-utilities.

For M.S. level with zero years of professional work experience, the average annual salary is $\$ 38,800$ for utilities and $\$ 40,100$ for non-utilities.

For Ph.D. level with zero years of professional work experience, the average annual salary is $\$ 41,500$ for utilities and $\$ 49,100$ for non-utilities.

\section{Nuclear engineers' salary comparisons between utilities and non-utilities}

Utilities in comparison to non-utilities tend to pay relatively more for B.S. level personnel and have larger salary differentials for years of experience within degree levels.

Non-utilities in comparison to utilities tend to pay relatively more for M.S. and Ph.D. level personnel and have larger salary differentials between degree levels for equal years of experience.

\section{Salaries for Health Physicists (see Tables 2 and 3)}

Health physicists' starting salaries reported by utilities and non-utilities

For B.S. degree with zero years of professional work experience, the average annual salary is $\$ 39,400$ for utilities and $\$ 34,500$ for non-utilities.

For M.S. degree with zero years of professional work experience, the average annual salary is $\$ 41,700$ for utilities and $\$ 39,500$ for non-utilities.

For Ph.D. degree with zero years of professional work experience, the average annual salary is $\$ 44,700$ for utilities and $\$ 48,300$ for non-utilities.

\section{Health physicists' salary comparisons between utilities and non-utilities}

Utilities in comparison to non-utilities tend to pay relatively more for B.S. and M.S. level personnel. Non-utilities in comparison to utilities tend to pay relatively more for Ph.D. level personnel, and have larger salary differences both for years of experience within degree levels and for degree levels for equal years of experience.

\section{Percentage Change Trends for Salaries, 1992-1996 (see Table 4)}

[These changes are for organizations that provided data in each pair of years being compared.]

The percentage increases in average starting salaries from 1995 to 1996, for both nuclear engineers and health physicists, are slightly higher than, or equal to, the annual increases recorded for 1994 to 1995 for all but B.S. level health physicists.

For B.S. and M.S. nuclear engineers the 1995 to 1996 salary changes are about the average of the annual changes that occurred between 1992 and 1995.

Ph.D. nuclear engineers had the highest percentage increase from 1995 to 1996 reported over the last 4 years.

The percentage change in health physicists' starting salaries from 1995 to 1996, for all degree levels, are lower (often considerably lower) than the annual increases reported between 1992 and 1994. 


\section{Table 2. Utilities Salary Information}

July 1996

\section{NUCLEAR ENGINEERS}

Annual Salary (rounded to nearest hundred)

\begin{tabular}{|c|c|c|c|c|c|c|c|c|c|c|c|c|c|c|c|}
\hline \multirow[b]{2}{*}{ Years of Experience: } & \multicolumn{5}{|c|}{ B.S. Degree Level Personnel } & \multicolumn{5}{|c|}{ M.S. Degree Level Personnel } & \multicolumn{5}{|c|}{ Ph.D. Degree Level Personnel } \\
\hline & Years & 1 Year & 3 Years & 4.7 Yrs & $\underline{8-10 Y r s}$ & 0 Years & 1 Year & $\underline{3 \text { Years }}$ & $4-7 \mathrm{Yrs}$ & $8-10 Y r s$ & 0 Years & 1 Year & 3 Years & $4-7 Y_{r s}$ & $\underline{8-10 Y r s}$ \\
\hline & 20 & 17 & & 12 & & & 14 & 13 & 7 & 7 & 8 & 8 & 8 & 4 & 5 \\
\hline Average ( & 7,10 & 39,000 & $\$ 44,900$ & 3,300 & & 800 & $\$ 40,100$ & 43,900 & 50,300 & $\$ 59$ & & $\$ 43,100$ & $\$ 45,000$ & $\$ 49,300$ & \\
\hline dian: & $\$ 37,100$ & $\$ 38,400$ & $\$ 44,200$ & $\$ 53,300$ & $\$ 61,800$ &, 300 & $\$ 40,100$ & $\$ 44,700$ & $\$ 51,000$ & $\$ 63$, & & $\$ 42,800$ & $\$ 44,800$ & 300 & \\
\hline Standard Deviation: & $\$ 2,500$ & 100 &, 700 & 100 & & & $\$ 2,600$ & $\$ 2,500$ & $\$ 4,200$ & & 100 & $\$ 4,400$ & $\$ 5,400$ & $\$ 5,100$ & $\$ 22,700$ \\
\hline Hiah: & $\$ 1,000$ & $\$ 45,700$ & 1,200 & $\$ 61,100$ & & 300 & $\$ 43,900$ & $\$ 48,000$ & 56,100 & $\$ 71$, & 7,100 & $\$ 48,500$ & $\$ 52,100$ & $\$ 54,800$ & $\$ 105,500$ \\
\hline Low: & $\$ 31,600$ & $\$ 33,800$ & $\$ 38,200$ & $\$ 42,600$ & $\$ 48,100$ & $\$ 33,800$ & $\$ 36,100$ & $\$ 39,700$ & $\$ 43,500$ & $\$ 49,000$ & $\$ 36,200$ & $\$ 36,200$ & $\$ 36,200$ & $\$ 43,900$ & $\$ 49,500$ \\
\hline
\end{tabular}

\section{HEALTH PHYSICISTS}

\begin{tabular}{|c|c|c|c|c|c|c|c|c|c|c|c|c|c|c|c|}
\hline \multirow[b]{3}{*}{ Years of Experience: } & \\
\hline & \multicolumn{5}{|c|}{ B.S. Degree Level Personnel } & \multicolumn{5}{|c|}{ M.S. Degree Level Personnel } & \multicolumn{5}{|c|}{ Ph.D. Degree Level Personnel } \\
\hline & 0 Years & 1 Year & 3 Years & $4.7 \mathrm{Yrs}$ & $\underline{8-10 \mathrm{Yrs}}$ & 0 Years & 1 Year & 3 Years & $4-7 \mathrm{Yrs}$ & 8 -10Yrs & 0 Years & 1 Year & 3 Years & $4-7 Y_{r s}$ & $8.10 \mathrm{Yrs}$ \\
\hline total received: & 13 & 12 & 13 & 6 & 6 & 10 & 9 & 11 & 3 & 3 & 9 & 9 & 9 & 2 & 2 \\
\hline Average (Mean): & $\$ 39,400$ & $\$ 41,500$ & $\$ 48,600$ & $\$ 54,500$ & $\$ 60,400$ & $\$ 41,700$ & $\$ 44,300$ & $\$ 49,100$ & $\$ 51,200$ & $\$ 51,800$ & $\$ 44,700$ & $\$ 46,900$ & $\$ 50,300$ & $\$ 50,700$ & $\$ 55,300$ \\
\hline Median: & $\$ 37,900$ & $\$ 39,600$ & $\$ 48,500$ & $\$ 52,900$ & $\$ 61,100$ & $\$ 40,700$ & $\$ 42,600$ & $\$ 45,400$ & $\$ 52,700$ & $\$ 49,000$ & $\$ 44,800$ & $\$ 46,200$ & $\$ 50,000$ & $\$ 50,700$ & $\$ 55,300$ \\
\hline Standard Deviation: & $\$ 5,300$ & $\$ 6,000$ & $\$ 8,200$ & $\$ 11,400$ & $\$ 11,800$ & $\$ 5,000$ & $\$ 5,800$ & $\$ 8,900$ & $\$ 4,500$ & $\$ 6,300$ & $\$ 4,800$ & $\$ 5,600$ & $\$ 7,900$ & $\$ 5,700$ & $\$ 8,100$ \\
\hline High: & $\$ 47,100$ & $\$ 50,400$ & $\$ 61,900$ & $\$ 74,000$ & $\$ 76,900$ & $\$ 49,300$ & $\$ 52,700$ & $\$ 66,200$ & $\$ 54,800$ & $\$ 59,000$ & $\$ 51,400$ & $\$ 55,000$ & $\$ 62,100$ & $\$ 54,700$ & $\$ 61,000$ \\
\hline Low: & $\$ 31,400$ & $\$ 32,700$ & $\$ 38,000$ & $\$ 42,600$ & $\$ 47,300$ & $\$ 35,600$ & $\$ 37,900$ & $\$ 37,900$ & $\$ 46,200$ & $\$ 47,300$ & $\$ 37,900$ & $\$ 37,900$ & $\$ 37,900$ & $\$ 46,600$ & $\$ 49,500$ \\
\hline
\end{tabular}


Table 3. Non-Utilities Salary Information

July 1996

\section{NUCLEAR ENGINEERS}

Annual Salary (rounded to nearest hundred)

\begin{tabular}{|c|c|c|c|c|c|c|c|c|c|c|c|c|c|c|c|}
\hline \multirow[b]{2}{*}{ ears of Experience: } & \multicolumn{5}{|c|}{ B.S. Degree Level Personnel } & \multicolumn{5}{|c|}{ M.S. Degree Level Personnel } & \multicolumn{5}{|c|}{ Ph.D. Degree Level Personnel } \\
\hline & QYears & 1 Year & 3 Years & $4.7 \mathrm{Yrs}$ & $8-10$ Yrs & $\underline{0 \text { Years }}$ & 1 Year & 3 Years & $4-7$ Yrs & $8-10$ Yrs & 0 Years & 1 Year & 3 Years & $4-7$ Yrs & 8-10Yrs \\
\hline total received: & 33 & 35 & 39 & 32 & 32 & 29 & 28 & 31 & 24 & 25 & 23 & 21 & 25 & 19 & \\
\hline an): & $\$ 36,700$ & $\$ 38,600$ & $\$ 43,000$ & $\$ 47,000$ & $\$ 57,300$ & 40,100 & $\$ 42,400$ & $\$ 46,200$ & $\$ 52,000$ & $\$ 61,800$ & 9,100 & $\$ 50,800$ & $\$ 55,400$ & $\$ 61,000$ & 69,300 \\
\hline ledian: & $\$ 36,100$ & $\$ 37,600$ & $\$ 41$, & $\$ 45,600$ & $\$ 54,400$ & 40,400 & $\$ 42,000$ & $\$ 45,800$ & $\$ 50,400$ & $\$ 58,000$ & 51,400 & $\$ 52,000$ & 00 & $\$ 59,000$ & $\$ 70,000$ \\
\hline d Deviation: & 7,500 & $\$ 8,900$ & 000 & $\$ 4,400$ & $\$ 9,900$ & $\$ 4,000$ & $\$ 4,300$ & $\$ 4,800$ & $\$ 4,300$ & $\$ 10,4$ & $\$ 6,500$ & $\$ 6,600$ & $\$ 7,600$ & $\$ 7,000$ & $\$ 8,500$ \\
\hline & 2,800 & $\$ 83,200$ & 0 & 0,800 & $\$ 92,7$ & & $\$ 52,300$ & $\$ 60,000$ & $\$ 60, c$ & $\$ 92$, & 0 & $\$ 63,100$ & $\$ 70,000$ & $\$ 76,200$ & $\$ 89,000$ \\
\hline ow. & 7,000 & $\$ 28,800$ & $\$ 30,900$ & $\$ 38,000$ & $\$ 46,800$ & $\$ 32,000$ & $\$ 34,000$ & $\$ 36,000$ & $\$ 45,000$ & $\$ 49,200$ & $\$ 41,200$ & $\$ 43,000$ & $\$ 44,800$ & $\$ 52,800$ & 55,200 \\
\hline
\end{tabular}

\section{HEALTH PHYSICISTS}

\begin{tabular}{|c|c|c|c|c|c|c|c|c|c|c|c|c|c|c|c|}
\hline \multirow[b]{3}{*}{ Years of Experience: } & \multirow{2}{*}{\multicolumn{5}{|c|}{ B.S. Degree Level Personnel }} & \multirow{2}{*}{\multicolumn{5}{|c|}{ M.S. Degree Level Personnel }} & \multirow{2}{*}{\multicolumn{5}{|c|}{ Ph.D. Degree Level Personnel }} \\
\hline & & & & & & & & & & & & & & & \\
\hline & O Years & 1 Year & 3 Years & $4-7$ Yrs & $8-10$ Yrs & 0 Years & 1 Year & 3 Years & 4-7Yrs & $8-10 Y_{r s}$ & 0 Years & 1 Year & 3 Years & $4.7 \mathrm{Yrs}$ & $8-10$ Yrs \\
\hline total received: & 28 & 32 & 32 & 33 & 30 & 24 & 26 & 25 & 23 & 24 & 18 & 19 & 20 & 17 & 20 \\
\hline Average (Mean): & $\$ 34,500$ & $\$ 36,600$ & $\$ 41,200$ & $\$ 46,500$ & $\$ 53,400$ & $\$ 39,500$ & $\$ 40,900$ & $\$ 45,000$ & $\$ 51,000$ & $\$ 59,900$ & $\$ 48,300$ & $\$ 49,600$ & $\$ 55,000$ & $\$ 61,000$ & $\$ 67,400$ \\
\hline Median: & $\$ 35,900$ & $\$ 37,100$ & $\$ 40,300$ & $\$ 47,000$ & $\$ 54,000$ & $\$ 39,700$ & $\$ 40,700$ & $\$ 45,500$ & $\$ 50,700$ & $\$ 59$, & $\$ 46,800$ & $\$ 47,800$ & $\$ 52,100$ & $\$ 63,000$ & $\$ 70,000$ \\
\hline Standard Deviation: & $\$ 5,700$ & $\$ 8,100$ & 1,200 & $\$ 5,500$ & $\$ 5,600$ & $\$ 4,400$ & $\$ 4,400$ & $\$ 5,400$ & $\$ 5,100$ & $\$ 9,900$ & $\$ 6,700$ & $\$ 6,900$ & $\$ 6,800$ & $\$ 7,500$ & $\$ 9,200$ \\
\hline High: & $\$ 52,000$ & $\$ 72,800$ & $\$ 83,200$ & $\$ 58,100$ & $\$ 65,800$ & $\$ 50,400$ & $\$ 51,600$ & $\$ 55,600$ & $\$ 58,800$ & $\$ 98,000$ & $\$ 60,200$ & $\$ 63,100$ & $\$ 68,900$ & $\$ 76,200$ & $\$ 86,300$ \\
\hline Low: & $\$ 22,000$ & $\$ 24,000$ & $\$ 30,000$ & $\$ 31,000$ & $\$ 43,100$ & $\$ 29,900$ & $\$ 32,400$ & $\$ 33,400$ & $\$ 41,400$ & $\$ 48,000$ & $\$ 40,300$ & $\$ 40,000$ & $\$ 45,800$ & $\$ 49,500$ & $\$ 53,000$ \\
\hline
\end{tabular}




\section{Table 4. Annual Salary Percentage Changes, 1992 - 1996}

For Personnel with Zero Years of Experience

The annual percentage changes are for organizations providing salary data for both years in each column.

FOR ALL ORGANIZATIONS (utilities plus non-utilities)

B.S. Level Personnel

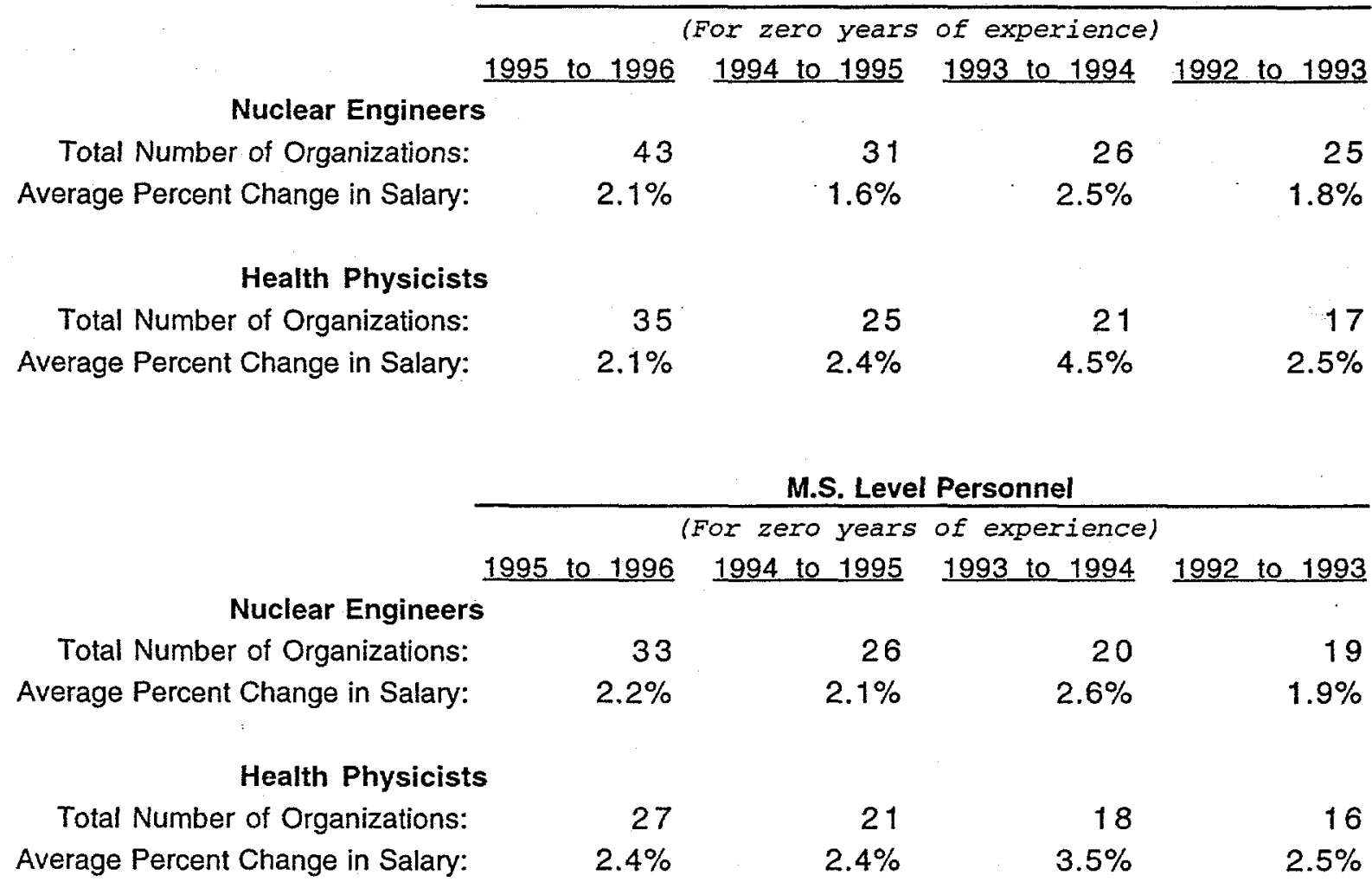

Ph.D. Level Personnel

\begin{tabular}{|c|c|c|c|c|}
\hline & \multirow{2}{*}{\multicolumn{4}{|c|}{ (For zero years of experience) }} \\
\hline & & & & \\
\hline & 1995 to 1996 & 1994 to 1995 & 1993 to 1994 & 1992 to 1993 \\
\hline \multicolumn{5}{|c|}{ Nuclear Engineers } \\
\hline & 23 & 19 & 11 & 12 \\
\hline Change in Salary: & $2.9 \%$ & $2.1 \%$ & $2.5 \%$ & $1.0 \%$ \\
\hline
\end{tabular}

Health Physicists

Total Number of Organizations: Average Percent Change in Salary:

$\begin{array}{rrrr}20 & 17 & 12 & 11 \\ 2.2 \% & 1.9 \% & 5.2 \% & 3.7 \%\end{array}$


FIGURES 
Figure 1. Nuclear Engineers' Salaries at Utilities, July 1996

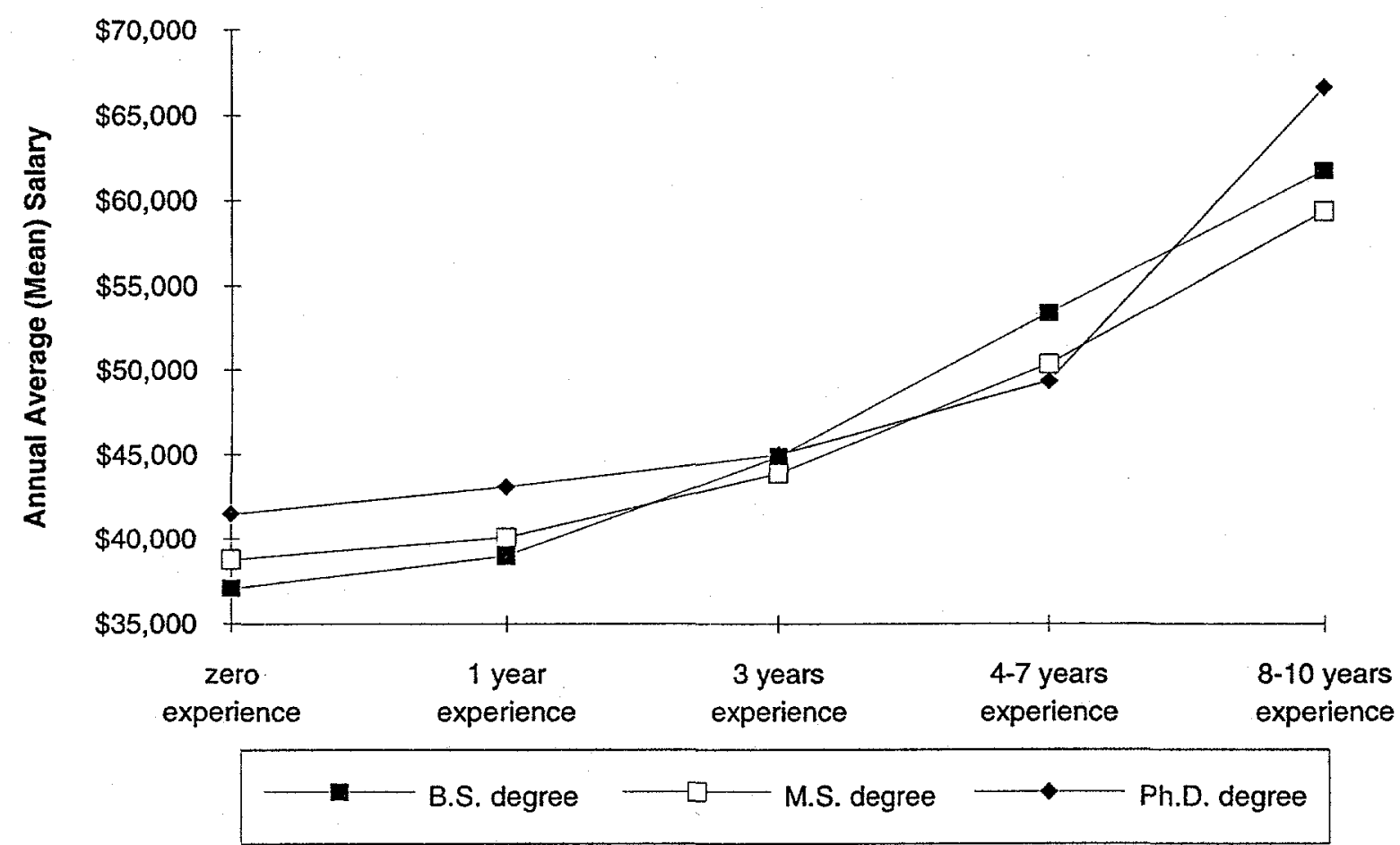


Figure 2. Nuclear Engineers' Salaries at Non-Utilities, July 1996

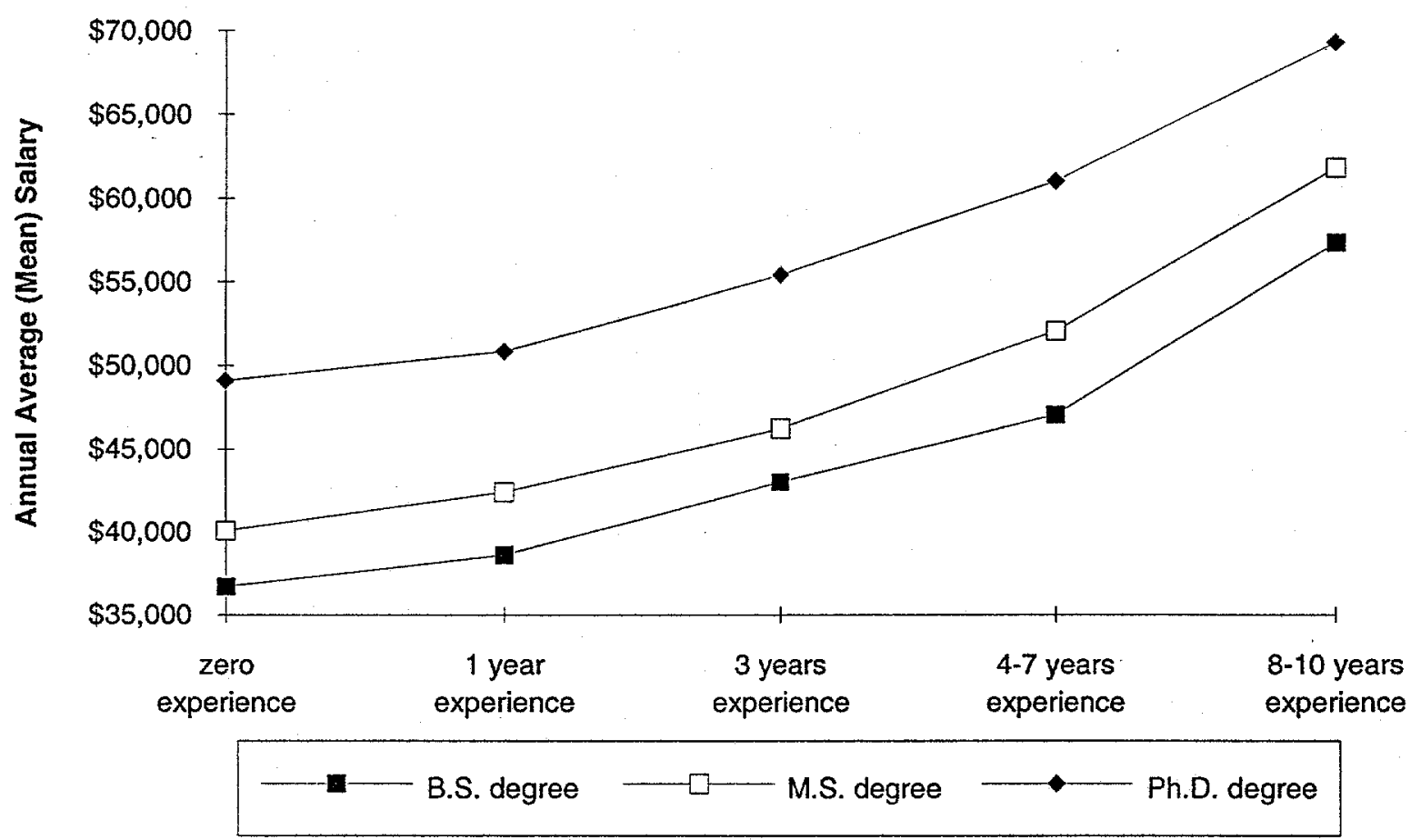


Figure 3. Health Physicists' Salaries at Utilities, July 1996

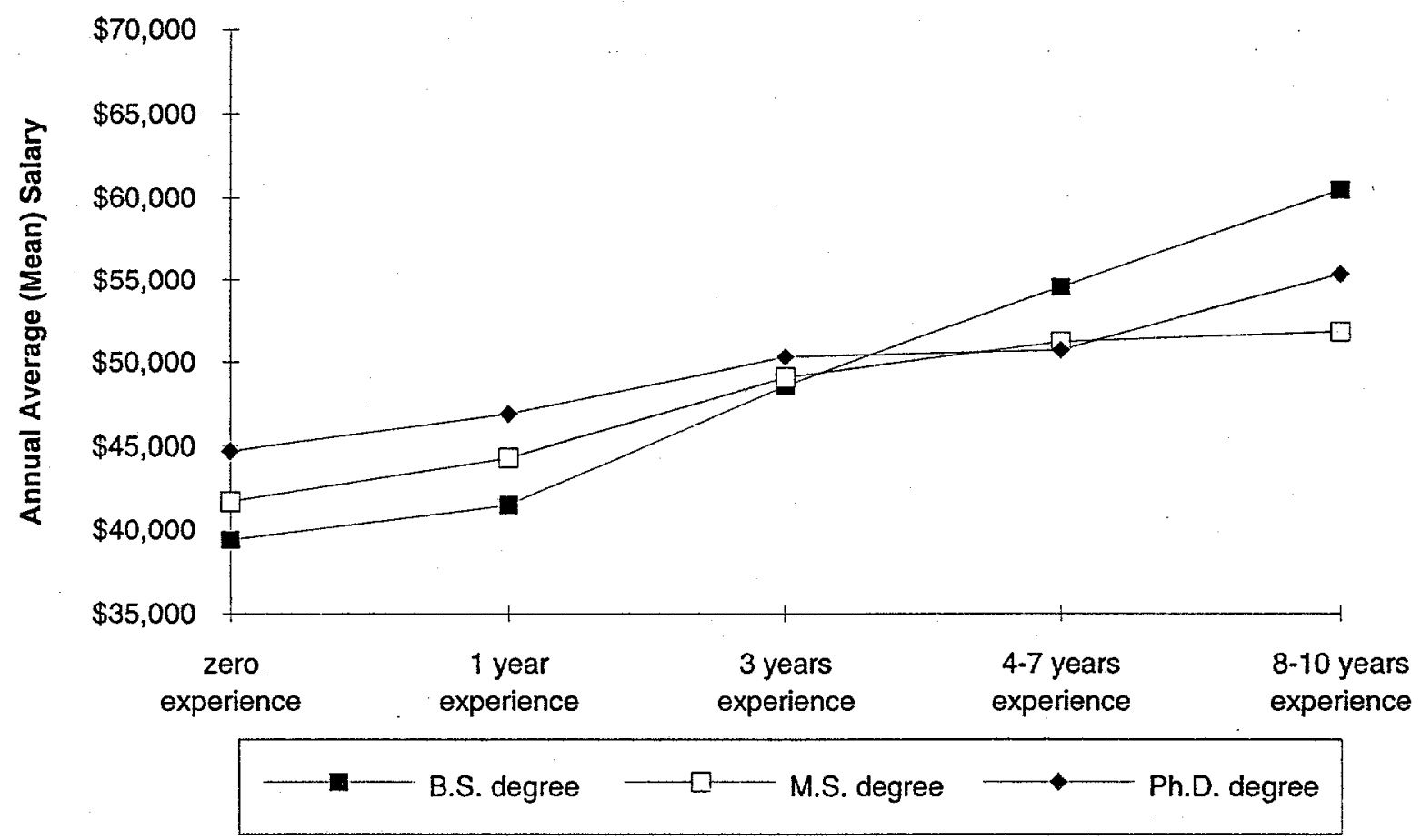


Figure 4. Health Physicists' Salaries at Non-Utilities, July 1996

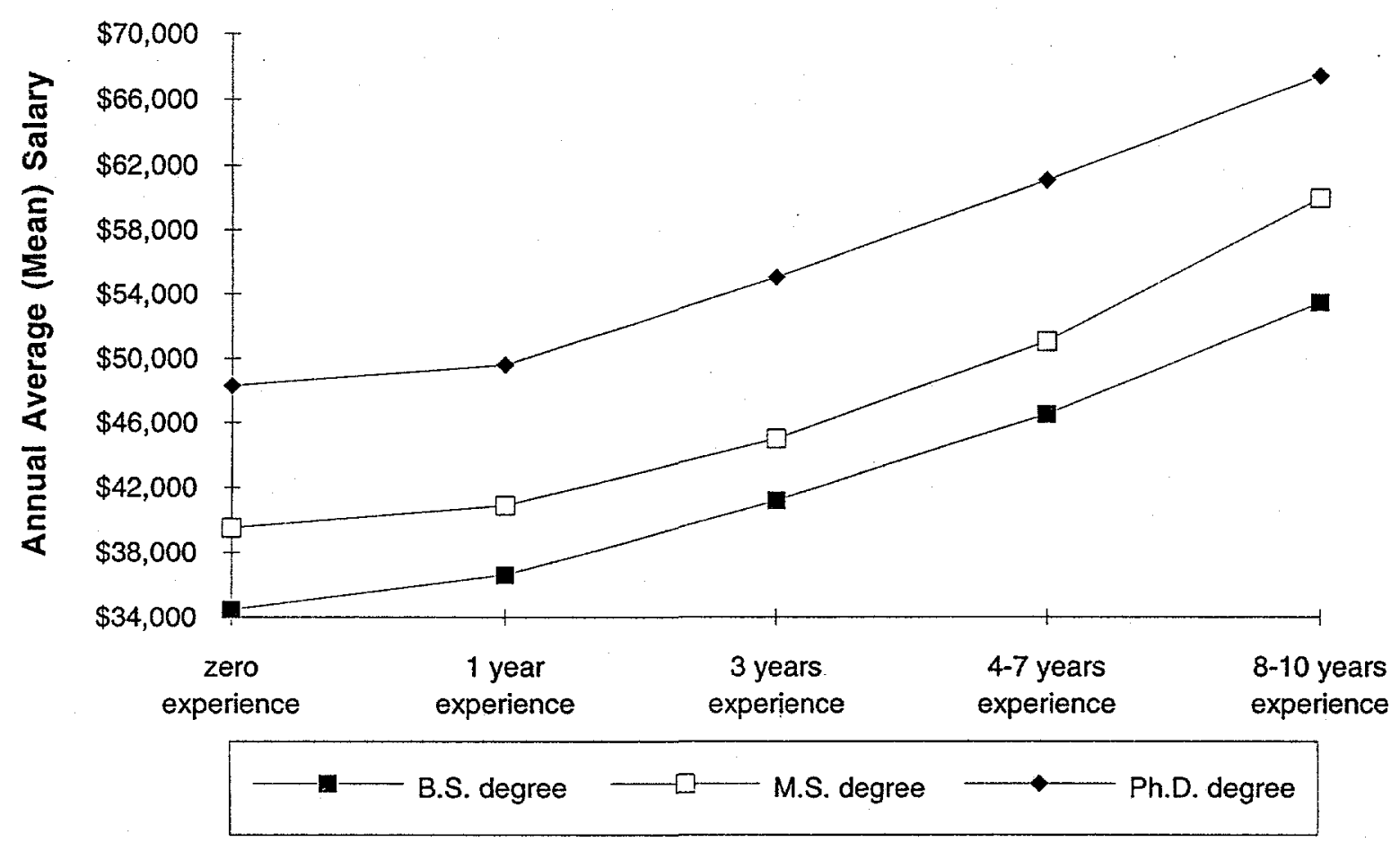


Figure 5. B.S. Level Personnel, Zero Years Experience, Annual Salary Changes, All Organizations (Utilities and Non-Utilities)

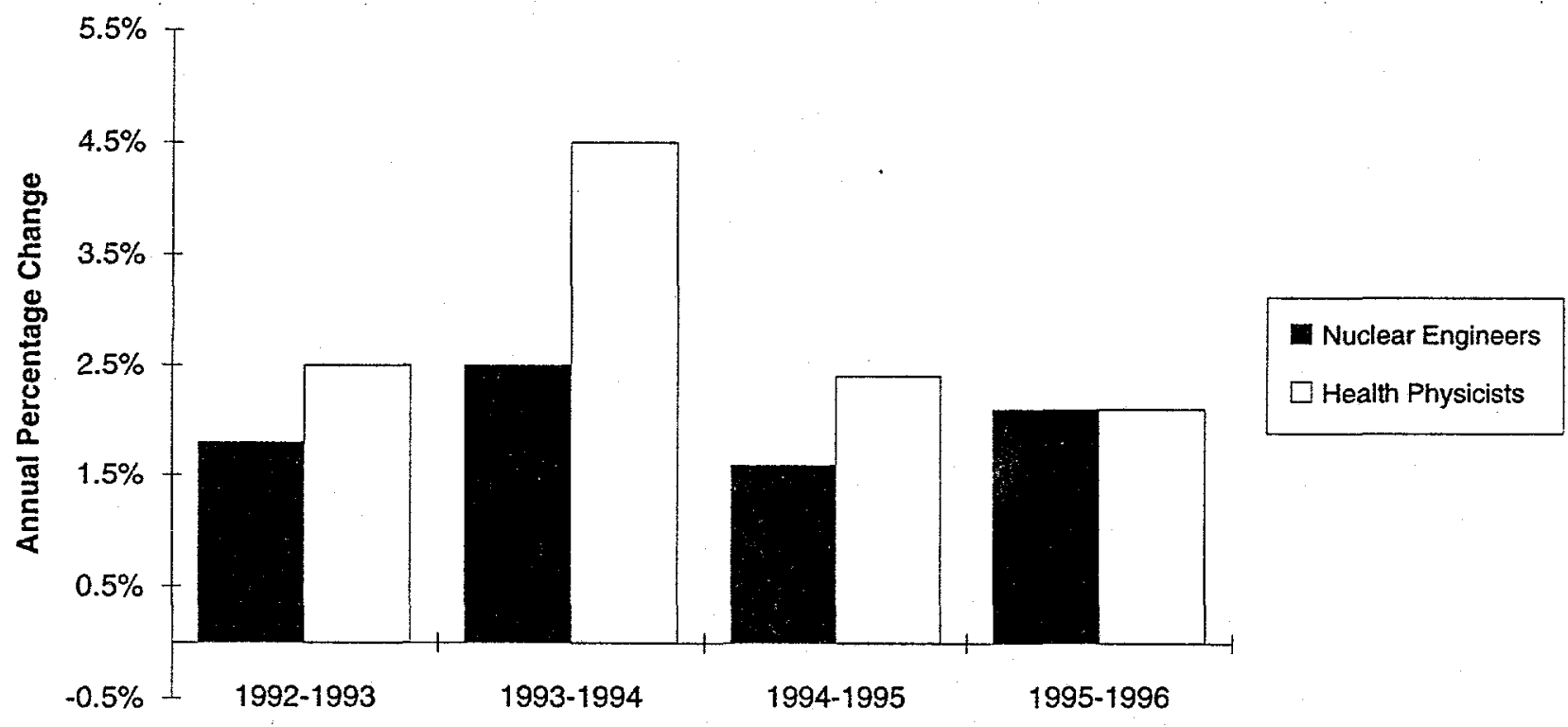

(For Organizations that Provided Data in Both Years Indicated) 
Figure 6. M.S. Level Personnel, Zero Years Experience, Annual Salary Changes, All Organizations (Utilities and Non-Utilities)

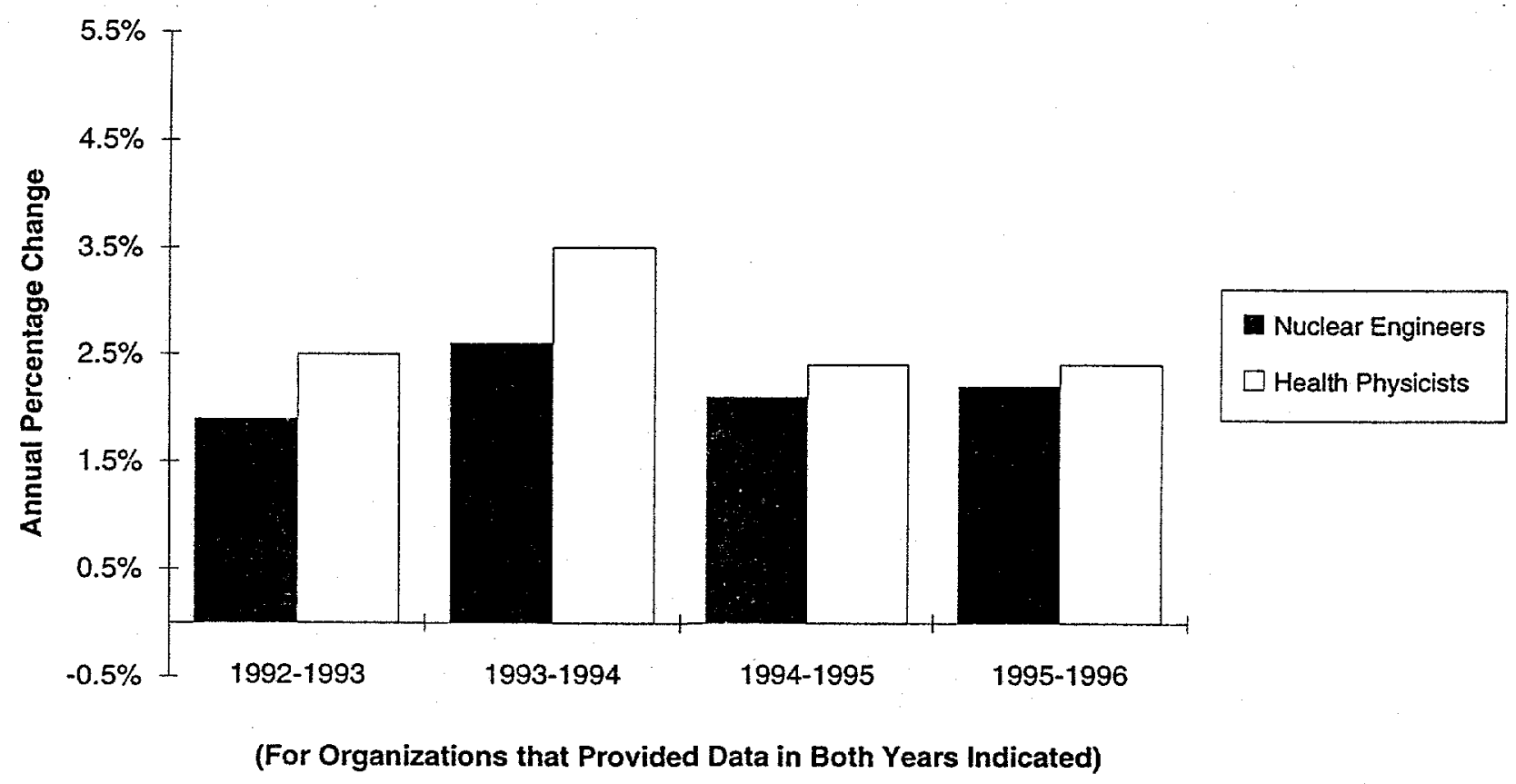


Figure 7. Ph.D. Level Personnel, Zero Years Experience, Annual Salary Changes, All Organizations (Utilities and Non-Utilities)

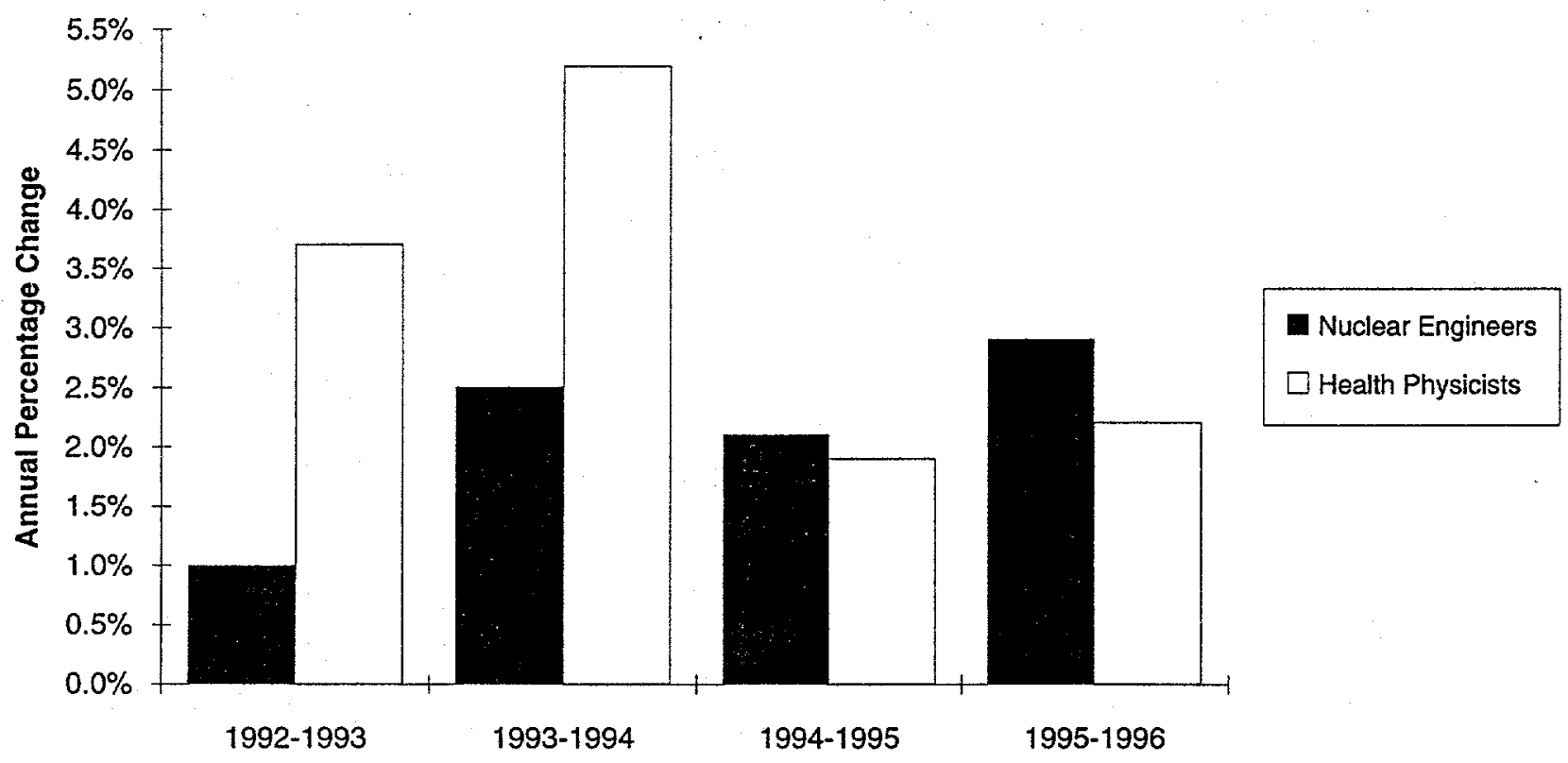

(For Organizations that Provided Data in Both Years Indicated) 


\section{SURVEY RESPONDENTS}

(Organizations that authorized listing of company name.)

\section{$\underline{\text { Utilities }}$}

Arizona Public Service

Centerior Energy Corporation

ComEd

Consolidated Edison Company of New York

Consumers Power Company

Duke Power Company

Duquesne Light Company

Entergy Services, Inc.

Florida Power Corporation

Houston Lighting and Power Company

Maine Yankee Atomic Power Company

Omaha Public Power District

PECO Energy Company

Tennessee Valley Authority

Texas Utilities Electric Company

The Southern Company

Vermont Yankee Nuclear Power

Virginia Power Company

Washington Public Power Supply System

Wisconsin Public Service Corporation
Non-Utilities

Abbott Laboratories

Allied Signal, Inc.

Analysis and Measurement Services Corporation

Argonne National Laboratory

Argonne National Laboratory-West

Babcock \& Wilcox NNFD

Battelle Pacific Northwest National Laboratory

Brookhaven National Laboratory

BW/IP International, Inc.

Computer Simulation and Analysis, Inc.

Duke Engineering \& Services

DuPont MERCK Pharmaceuticals

Electric Power Research Institute

Frametone Technologies

Hagler Bailly Consulting

Halliburton Energy Services

Interem, Inc.

International Technology Corporation

Interstate Nuclear Services Corporation

JBF Associates, Inc.

Knolls Atomic Power Laboratory

Lockheed Martin Energy Systems

Lockheed Martin Energy Research Corp.

Lockheed Martin Idaho Technologies

Lockheed Martin Utility Services

Los Alamos National Laboratory

Los Alamos Technical Associates, Inc.

Mason \& Hanger - Silas Mason Company, Inc.

NAC International

Nuclear Fuel Services, Inc.

Oak Ridge Associated Universities, Inc.

Pickard, Lowe, \& Garrick, Inc.

Plant Technical Services

Radiation Consulting Associates

Rogers \& Associates Engineering Corporation

RSA Laboratories, Inc.

Rust Geotech, Inc.

Sandia National Laboratories

Science Applications International Corporation

Scientech, Inc.

Shonka Research Associates

Southeastern Universities Research Association

Stanford Linear Accelerator Center

Utility Resource Associates

West Valley Nuclear Services Company

Westinghouse Electric-Bettis Atomic Power Lab.

Westinghouse Hanford Company

Westinghous Electric Corp-Naval Reactor Facility 